\title{
"Standardless" Quantitative Electron Beam X-ray Microanalysis: The Situation Remains caveat emptor!
}

\author{
Dale E. Newbury
}

National Institute of Standards and Technology, Gaithersburg, MD 20899-8371

In 1999 MAS celebrated the 50 ${ }^{\text {th }}$ anniversary of Prof. Raymond Castaing's first conference presentation describing his electron probe microanalyzer (EPMA), the subject of his thesis.[1,2] The work of the EPMA community over five decades has given us a robust microanalytical technique capable of a high degree of accuracy, with $95 \%$ of analyses falling within $\pm 5 \%$ relative when materials of known, multi-element composition are tested. The fundamental principle of this method is the comparison of the unknown spectrum to that of one or more standards obtained under identical conditions of beam energy, electron incidence angle, spectrometer take-off angle and efficiency, and calibrated electron dose. Matrix (inter element) effects between the unknown composition and that of the standards are calculated as a series of correction factors by various schemes.[3] The standards required can be as simple as pure elements, or binary compounds for elements that are incompatible with instrument vacuum conditions or which are unstable under the beam (e.g., $\mathrm{FeS}_{2}$ can be chosen for $\mathrm{S}$ instead of pure $\mathrm{S}$, which degrades). The standards/matrix corrections method gives a large measure of "analytical flexibility", which can be defined as the capability of dealing with unknowns of complex composition with a simple standards suite, thus eliminating the need to resort to standards that closely resemble the unknowns in composition.

Despite the robust performance of the Castaing standards/matrix corrections method, and its relatively rapid speed and ease of operation when stored spectra are used in energy dispersive x-ray spectrometry (EDS), an ever increasing fraction of the analyses performed by the SEM community is done with "standardless" analysis procedures.[4,5] Standardless analysis as a term covers a suite of methods in which only the spectrum of the unknown is required for the analysis. The needed standard intensities are supplied by some type of a model, ranging from a pure "first principles" approach, based upon theoretical descriptions of x-ray generation, propagation, and detection, to mathematical fits of measured standards data ("fitted standards" or "remote standards"). Only beam energy, spectrometer take-off angle, and window parameters are required. Electron dose dependence is eliminated through internal normalization, so results always total $100 \%$. The attraction of the standardless approach is obvious. The SEM user can obtain a "quantitative analysis" with virtually no effort and with apparently trivial quality assurance demands.

What are the consequences of using standardless analysis? When tested against known multicomponent materials, especially those involving mixtures of elements that require selection of x-ray peaks from $\mathrm{K}, \mathrm{L}$ and $\mathrm{M}$ shells, the relative errors observed with standardless analysis are much greater than the $\pm 5 \%$ error range (for $95 \%$ of analyses) achieved with the standards/matrix correction method.[4,5] An error distribution for the earliest "first principles" standardless formulation (the "standardless miracle" developed by Fiori and Swyt [6]) showed an error range of $\pm 50 \%$ relative for $95 \%$ of analyses.[4] Tests of the error distributions for two commercial standardless procedures based on variants of the fitted (or remote) standards approach showed error ranges such that $95 \%$ of analyses fell within approximately $\pm 25 \%$ relative error.[5] 
Can the standardless analysis situation be improved? The early first principles approach of Fiori and Swyt used the available physical parameters from the literature, many of which were recognized to have large uncertainties, e.g., especially ionization cross sections and fluorescence yields for the L- and M-shells.[6] Systematic efforts at examining and improving the theoretical basis for standardless analysis have been reported recently.[7] A spectrum simulation scheme based on improved descriptions of both characteristic and continuum x-rays in which the peak-tobackground parameter plays an important role has been described.[8] In this simulation, which can form the basis for a new standardless analysis procedure, an RMS error of 7.1\% was achieved in a comparison with a large database of experimentally measured peak intensities. Studies such as these suggest that "help is on the way." A robust standardless analysis procedure that is thoroughly tested against well characterized spectral databases, similar to the traditional tests of matrix correction procedures, will be of great value.

However, the current standardless analysis situation remains "caveat emptor." The user of standardless analysis is frequently confronted with a "black box" software procedure, the details of which may not be explained and often not supported by extensive testing. Analysts are thus left to perform the necessary tests on their own microhomogeneous materials to assess accuracy. Since such materials are often difficult to find, this may require careful selection of alternative materials that at least contain the elements of interest, even if at much different concentrations. A good test is to see if the procedure gives the same results, within a reasonable error, as the beam energy is changed. Moreover, adapting a standardless analysis procedure to a particular instrument is critically dependent on accurately describing the efficiency of the EDS. While this can certainly be done, the user must be aware of detector "aging", usually due to ice accumulation, which can affect the low photon energy efficiency $(<3 \mathrm{keV})$. With SEM specimens and scanning procedures, the analysis point may be selected anywhere in the scan field so that the x-ray source moves relative to the EDS. Since atmosphere-supporting thin windows are supported by a grid, the exact point of origin of the x-ray source can affect the shadowing of the detector, and thus its efficiency. A change in total efficiency would normalize out, but any differential effects with photon energy would be manifest as another source of analytical errors.

\section{References}

[1] R. Castaing (1951) thesis, Univ. of Paris.

[2] R. Marinenko, ed. 50 Years of EPMA, Micros. Microanal. 7(2001) 94.

[3] J.I. Goldstein et al., Scanning Electron Microcopy and X-ray Microanalysis, Plenum, New York, 1992, 417.

[4] D.E. Newbury et al., Analytical Chem., 67 (1995) 1866.

[5] D.E. Newbury, Micros. Microanal., 4 (1999) 585.

[6] C.E. Fiori and C.R. Swyt, Microbeam Analysis, San Francisco Press, 1989, 236.

[7] J. Wernisch and K. Rohrbacher, Mikrochim. Acta [Suppl], 15 (1998) 307.

[8] P. Duncumb et al. Micros. Microanal, 7 (2001) 341. 